\title{
A Depressão Materna e o Comportamento de Crianças em Idade Escolar ${ }^{1}$
}

\author{
Luciana Mian \\ Louise Azenha Tango \\ Juliana Lopes \\ Sonia Regina Loureiro ${ }^{2}$ \\ Universidade de São Paulo
}

\begin{abstract}
RESUMO - A depressão materna caracteriza-se como condição de vulnerabilidade ao desenvolvimento infantil. No presente estudo, objetivou-se comparar o perfil comportamental, as percepções e os eventos de vida de escolares que convivem com a depressão materna (G1) aos daqueles que convivem com mães sem história psiquiátrica (G2), segundo as informações obtidas com as mães e as crianças. Avaliou-se 40 crianças, de 7 a 12 anos, por meio do Teste Raven, da Escala Infantil Piers-Harris de Autoconceito e da Entrevista de Eventos Vitais. As mães foram avaliadas pela Entrevista Clínica Estruturada para o DSM-IV para a confirmação diagnóstica, e responderam a Escala Comportamental Infantil A2 de Rutter. A depressão materna mostrouse associada a problemas comportamentais das crianças, segundo o relato das mães e a percepção das crianças.
\end{abstract}

Palavras-chave: depressão materna; criança; problemas de comportamento; autoconceito; eventos de vida.

\section{Maternal Depression and School-Aged Children Behavior}

\begin{abstract}
Maternal depression is characterized as a condition of vulnerability to children's development. The aim of this study was to compare the behavioral profile, the self-perceptions and the life events experienced by school-aged children that live with maternal depression (G1) to those of children who live with mothers without psychiatric history (G2), according to mothers' and children's perceptions. Forty children, from 7 to 12 years old, were evaluated by the Raven Test, the Piers-Harris Children's Self-Concept Scale and the Interview of Vital Events. Mothers were evaluated by the Structured Clinical Interview for DSM-IV (SCID) for diagnostic confirmation, and answered to the Children's Behavioral Scale A2 by Rutter. Maternal depression was associated with behavioral problems according to mothers' and children's perceptions.
\end{abstract}

Keywords: maternal depression; child; behavior problems; self-concept; life events.

O interesse pelo impacto dos problemas de saúde mental na vida humana é crescente, em função da dimensão epidemiológica dos transtornos e pelos tipos de prejuízos associados, envolvendo o indivíduo, a família e a comunidade. Os levantamentos epidemiológicos populacionais realizados no Brasil apontam para uma prevalência aproximada de transtornos mentais de $30 \%$ na população adulta, sendo que $20 \%$ desses indivíduos identificados precisarão de atenção especializada em serviços de saúde mental; esses levantamentos apontam, ainda, que para a população feminina há um maior predomínio dos transtornos depressivos (Mari \& Jorge, 2002).

Os transtornos depressivos atingem de $10 \%$ a $20 \%$ de mulheres em idade fértil, mães e de baixa renda, pelo menos uma vez na vida, e para aproximadamente um terço dessas mulheres os sintomas depressivos são recorrentes e persistem por toda a vida (Kendler \& Prescott, 1999).

Sob a perspectiva teórica da psicopatologia do desenvolvimento, a depressão materna tem sido considerada uma situação pouco favorecedora do desenvolvimento infantil, podendo se configurar em um evento estressante e, consequentemente, em uma situação de risco (Boyle \& Pickles, 1997; Goodman \& Gotlib, 1999; Langrock, Compas, Keller,

1 Apoio financeiro: CNPq.

2 Endereço: Rua Anita Garibaldi, 1419. Ribeirão Preto, SP. CEP 14085480.E-mail: srlourei@fmrp.usp.br.
Merchant \& Copeland, 2002; Luoma \& cols., 2001; Sameroff \& Fiese, 2005; Wright \& Masten, 2006; Yunes \& Szymanski, 2001).

Os efeitos adversos da depressão materna podem ser verificados pelo impacto de tal condição nos indicadores de adaptação da criança, dentre os quais se destaca o desempenho frente a tarefas de desenvolvimento típicas do período de vida em que a criança se encontra. Tais tarefas envolvem os domínios físico, emocional, cognitivo, moral e comportamental e são referentes às expectativas de realização e desempenho, próprias de cada sociedade, em um dado contexto histórico (Wright \& Masten, 2006). Para as crianças em idade escolar, as principais tarefas de desenvolvimento a serem cumpridas referem-se ao desempenho acadêmico e à socialização e se expressam pelo ajustamento ao ambiente escolar, pela capacidade de se dar bem com os companheiros e pela conduta pró-social. Por meio do cumprimento dessas tarefas a criança se mostraria competente e possuidora da capacidade de produção e realização (Marturano \& Loureiro, 2003).

Com relação às mulheres/mães com depressão, características próprias do quadro depressivo, como os afetos negativos e imprevisíveis, expressos por irritação e tristeza, e os comportamentos de suporte reduzidos, como elogiar, cuidar e dar carinho, dificultam a provisão das necessidades emocionais e sociais dos filhos, influenciando a qualidade da interação e a organização do ambiente familiar e favorecendo 
os problemas comportamentais das crianças (Goodman \& Gotlib, 1999; Langrock \& cols, 2002).

O quadro depressivo materno favorece o afastamento da mãe com relação à rotina diária da família, quase sempre com a falta de suporte adequado à criança, podendo não fornecer o apoio e as manifestações afetivas suficientes para o desenvolvimento pleno das capacidades e habilidades esperadas das crianças, em diversos domínios. Em geral, os filhos de mães com história de depressão apresentam mais problemas escolares, menor competência social, baixos níveis de autoestima e elevados níveis de problemas de comportamento (Cummings, 1995).

Goodman e Gotlib (1999), ao sugerir que múltiplos mecanismos agem moderando ou mediando os riscos desenvolvimentais a que estão expostas crianças que convivem com a depressão materna, questionam se a possível hereditariedade da depressão favoreceria mecanismos neuroreguladores disfuncionais inatos e se os comportamentos e cognições maternas negativas implicariam em um contexto estressante de vida para a criança. Assim, segundo esses autores, a transmissão do risco estaria associada à emergência de vulnerabilidades no âmbito psicobiológico (do sistema nervoso central, por exemplo), cognitivo (como baixa auto-estima e cognições disfuncionais), afetivo (incluindo baixa tolerância e dificuldades de controle emocional) e comportamental ou interpessoal (habilidades sociais inadequadas, problemas na concentração, controle de impulso inadequado).

Diversos estudos que avaliaram o impacto da depressão materna para o comportamento da criança constataram que há maior probabilidade de as crianças filhas de mães com depressão, em comparação a crianças que convivem com mães sem história psiquiátrica, apresentarem problemas comportamentais, de externalização e internalização. (Boyle \& Pickles, 1997; Langrock \& cols., 2002; Najman \& cols., 2001). Langrock e cols., por exemplo, relataram que crianças que convivem com depressão na família apresentam mais riscos para problemas internalizantes, como ansiedade e depressão, e problemas externalizantes, como agressividade, quando comparadas à população normativa. Esses autores também constataram que o relato de estresse e depressão, por parte das mães, quando seus filhos tinham cinco anos, foi preditor de problemas de comportamento para tais crianças aos sete anos. Najman e cols. relataram que quanto mais sintomas de ansiedade e depressão a mãe apresenta, mais problemas internalizantes e externalizantes são relatados com relação a seus filhos, predominando as manifestações internalizantes para as meninas e as externalizantes para os meninos. Boyle e Pickles, por sua vez, relataram que a depressão materna mostrou-se associada significativamente a problemas de hiperatividade para as crianças de cinco a 12 anos, e na faixa etária de 12 a 16 anos associou-se significativamente à desordem de conduta.

Luoma e cols. (2001) examinaram a associação entre sintomas depressivos maternos e a presença de problemas emocionais e comportamentais das crianças, em diferentes estágios de desenvolvimento, constatando que as mães sintomáticas, comparativamente às não sintomáticas, relataram mais problemas de comportamento e de baixa competência com relação aos seus filhos, nas diferentes fases do desenvolvimento. De modo semelhante, Weissman e Jensen (2002) relataram que mães com depressão referiram que seus filhos apresentavam mais problemas emocionais que as crianças em geral, e ainda, que elas experimentavam conflitos frequentes com estes em suas práticas educativas.

A visão negativa que as mães depressivas têm de seus filhos, como por exemplo, a atribuição de baixa competência às crianças, pode influenciar também as percepções que estas têm sobre si, influenciando o seu autoconceito. Harter (1996) ressalta que a construção do autoconceito da criança está associada ao seu processo de desenvolvimento, às experiências sociais e as expectativas externas, ou seja, há uma internalização das opiniões dos outros. Assim sendo, a aprovação por parte dos pais e do ambiente social no qual a criança convive contribui de forma decisiva para o desenvolvimento da percepção que a criança tem de si, bem como do seu papel social no meio.

Considera-se, com base nas afirmativas prévias, que a convivência com a depressão materna pode constituir-se para as crianças em um evento estressor. Os eventos estressores são condições temporárias ou transitórias ligadas a eventos de vida. Uma grande variedade de eventos tem sido classificada como estressores, desde eventos maiores, como acontecimentos catastróficos e traumáticos, até pequenos acontecimentos do dia-a-dia, como as situações frustrantes e os desentendimentos com familiares e colegas (Pereira, 2001).

A depressão materna tem sido considerada um evento estressante diferenciado de outros que podem ocorrer na vida das crianças, pois quase sempre não se constitui em um evento único, dadas as elevadas taxas de reincidência como evolução característica desse quadro clínico. Dessa forma, muitas crianças que convivem com tal condição estão expostas a mais de um episódio depressivo materno, o que aumenta o risco de efeitos adversos associados à depressão materna (Goodman \& Gotlib, 1999).

Ressalta-se que os filhos de mães depressivas estão expostos não somente à depressão de suas mães, mas também a uma variedade de estressores associados que envolvem a discórdia familiar (tais como relações maritais tensas, hostis e conflituosas) e problemas ocupacionais, entre outros. A quantidade de eventos negativos associados com a depressão também pode explicar os efeitos adversos para as crianças. As crianças cujas mães depressivas relataram elevados níveis de eventos de vida estressantes nos domínios econômico, marital e das relações sociais, apresentaram mais problemas de ajustamento do que as crianças cujas mães depressivas relataram baixos níveis de eventos estressantes (Goodman \& Gotlib, 1999).

A depressão materna pode prejudicar, ainda, o desenvolvimento das habilidades das crianças no que diz respeito à autoregulação emocional. Tal prejuízo mostra-se associado à exposição a fatores de risco inter-relacionados, como o estresse crônico e os conflitos maritais, e às dificuldades de auto-regulação por parte das mães enquanto modelos de aprendizagem (Silk, Shaw, Skuban, Oland \& Kovacs, 2006). Destaca-se assim, a complexidade das questões envolvidas na influência da depressão materna para o desenvolvimento infantil, por envolver também aspectos relativos a outros estressores.

Um outro ponto que tem sido abordado com relação à depressão materna diz respeito a um aspecto metodológico 
dos estudos, os quais frequentemente tomam as mães como as únicas informantes sobre o comportamento dos seus filhos, o que, segundo Boyle \& Pickles (1997), pode favorecer o questionamento da confiabilidade de tal informação. Esse questionamento se daria em função de possíveis erros de relato decorrentes da sintomatologia depressiva. Nesse sentido, considerando o relato da mãe e o da própria criança, Kinsman e Wildman (2001) encontraram que as crianças filhas de mães com depressão se auto perceberam mais positivamente em relação ao seu funcionamento diário, comparativamente ao relato de suas mães. Corroborando tal achado, Chilcoat e Breslau (1997) observaram que, apesar de as mães com depressão acentuarem os problemas de seus filhos, essas avaliações não diferiram das realizadas pelos professores das crianças: em ambos os casos foi relatado que as crianças apresentavam um alto índice de problemas de externalização. A influência da depressão materna na avaliação comportamental que tais mães fazem de seus filhos foi também constatada em outros estudos que observaram que elas tendem a relatar mais problemas de comportamento com relação aos seus filhos do que fazem as próprias crianças ou seus professores, refletindo uma tendência das mães depressivas em acentuarem suas próprias dificuldades e os problemas de suas famílias (Boyle \& Pickles, 1997; Kinsman \& Wildman, 2001; Najman \& cols., 2001). Constata-se assim, a importância de se considerar dados de fontes independentes, entre outras, a perspectiva da própria criança sobre o seu próprio comportamento e os acontecimentos de sua vida diária.

Considerando o exposto, o presente estudo objetivou caracterizar o perfil comportamental, as autopercepções e os eventos de vida experimentados por crianças que convivem com a depressão materna, em comparação a crianças que experimentam o convívio com mães sem história psiquiátrica, tendo por fontes de informações as mães e as crianças.

\section{Método}

\section{Participantes}

Participaram do estudo 40 crianças, de ambos os sexos, residentes com suas mães biológicas, procedentes de famílias atendidas nos Núcleos do Programa de Saúde da Família do Centro de Saúde Escola da Faculdade de Medicina de Ribeirão Preto (FMRP-USP), ou famílias residentes na área compreendida pelos Núcleos.

As crianças foram distribuídas em dois grupos, a saber: G1 - constituído por 20 crianças, cujas mães apresentavam história clínica psiquiátrica com diagnóstico clínico de depressão; e G2 - constituído por 20 crianças, cujas mães não apresentavam história clínica psiquiátrica, recente ou pregressa. A partir das características demográficas das crianças de G1, buscou-se compor o grupo de comparação, G2. Foram excluídas do estudo crianças órfãs ou com história de adoção, com retardo mental, com história de doenças orgânicas crônicas ou com deficiências sensoriais aparentes.

A seleção dos participantes do estudo foi realizada tendo por base informações de 1.500 prontuários de mulheres na faixa etária de 25 a 40 anos, com filhos com idades entre sete e 12 anos, que apresentavam o diagnóstico clínico de depressão, com história de atendimento psiquiátrico ou semiinternação, nos últimos dois anos, estando em remissão dos sintomas há pelo menos seis meses. Todas as mães incluídas no G1 apresentavam diagnóstico de depressão moderada, ou grave, e no momento da coleta de dados não estavam sintomáticas e mantinham-se em seguimento psiquiátrico.

As seguintes categorias do CID-10 (Organização Mundial de Saúde, 1992/1993) foram incluídas como diagnóstico psiquiátrico de depressão: F 32.1 - Depressão Moderada, F 32.2 - Depressão Sem Sintomas Psicóticos, F 32.3 - Depressão com Sintomas Psicóticos e F33 - Depressão Recorrente. Foi considerado critério de exclusão o diagnóstico de Depressão Leve ou como comorbidade de outras doenças. As mães de ambos os grupos foram avaliadas sistematicamente por meio da Entrevista Clínica Estruturada para o DSM-IV (SCID I) e DSM-III-R (SCID II), com o objetivo de excluir outros diagnósticos, como comorbidade para o G1, e de garantir a ausência de história psiquiátrica recente e pregressa para o G2.

As crianças de G1 apresentavam média de idade de nove anos e quatro meses $(\mathrm{DP}=1,4)$ e as crianças de $\mathrm{G} 2$ tinham média de idade de 10 anos ( $\mathrm{DP}=1,7)$. Quanto ao nível cognitivo, avaliado por meio do Teste Raven, a média dos resultados obtidos por G1 e G2 foi, respectivamente, percentil 64,20 $(\mathrm{DP}=24,81)$ e $66,70(\mathrm{DP}=23,28)$. Com relação à escolaridade das crianças, G1 tinha uma média de 3,6 anos de escolaridade $(\mathrm{DP}=1,27)$, enquanto $\mathrm{G} 2$ apresentava média de 2,7 anos $(\mathrm{DP}=1,26)$. As mães de G1 apresentaram, em média, 6,55 anos de escolaridade ( $\mathrm{DP}=3,63)$, enquanto que as mães de $\mathrm{G} 2$ apresentaram, em média, 8,45 anos de escolaridade ( $\mathrm{DP}=4,47)$. Predominaram as configurações familiares caracterizadas por mães, pai/padrasto e irmãos. Com base em análise estatística, não foram observadas diferenças significativas quanto às variáveis relativas à idade, nível intelectual (avaliado pelo teste Matrizes Progressivas Coloridas de Raven - Escala Especial) das crianças, a escolaridade das mães e configurações familiares. Observou-se que as crianças de G1 apresentaram, com diferença significativa, maior escolaridade do que as crianças de G2, contudo não se constatou diferenças quanto a adequação da idade à série cursada.

O presente estudo foi apreciado e aprovado pelo Comitê de Ética do Centro de Saúde Escola da FMRP-USP (Protocolo $n^{\circ}$ 0011/CEP-CSE-FMRP-USP). A participação das crianças, bem como de suas mães, foi voluntária, mediante a assinatura do Termo de Consentimento Livre e Esclarecido por parte das mães.

\section{Instrumentos e materiais}

Com as mães foram utilizados os seguintes instrumentos:

- Entrevista Clínica Estruturada para o DSM-IV(SCID): traduzida e adaptada para o nosso meio por Del-Ben e cols. (2001). Foram utilizados a Revisão Geral e os módulos A e $\mathrm{B}$, visando à confirmação diagnóstica e as exclusões de comorbidade e de doença psiquiátrica.

- Escala Comportamental Infantil A2 de Rutter (ECI): validada e padronizada para escolares de Ribeirão Preto por 
Graminha (1994). Objetiva avaliar problemas emocionais e comportamentais da criança, sendo respondida pelas mães. A ECI inclui 36 itens, fornecendo escore global e escores parciais relativos a problemas de saúde (oito itens), hábitos (sete itens) e comportamento (21 itens).

Com as crianças, os seguintes instrumentos foram usados:

- Matrizes Progressivas Coloridas de Raven - Escala Especial: instrumento composto por 36 itens, divididos em três séries, o qual foi padronizado para a avaliação do nível intelectual das crianças brasileiras (Angelini, Alves, Custódio, Duarte \& Duarte, 1999).

- Escala Infantil Piers-Harris de Autoconceito: "O que eu sinto sobre mim mesmo" - proposta por Piers e Harris em 1984, e traduzida e adaptada para o nosso meio por Jacob (2001). Tal escala, respondida pelas crianças, tem o objetivo de avaliar seu autoconceito, sendo formada por 80 itens, os quais são apresentados sob a forma de sentenças. Tais sentenças avaliam o autoconceito global da criança e o autoconceito em seis subcategorias: comportamento (16 itens), status intelectual e acadêmico (17 itens), aparência (13 itens), ansiedade (14 itens), popularidade (12 itens) e felicidade e satisfação (10 itens).

- Entrevista de Eventos Vitais Positivos e Negativos: entrevista semiestruturada para investigar eventos de vida positivos e negativos, conforme proposição de Dell'Aglio (2000), e modificada por Bianchi (2002). Tal instrumento investiga eventos cotidianos que podem ocorrer na vida de crianças em idade escolar e que podem ser avaliados como positivos (ganhar presentes, ir bem na escola, entre outros) ou negativos (não poder fazer algo que queria muito, ficar doente, entre outros), envolvendo, quanto aos eventos positivos e negativos, três partes distintas: relato espontâneo, relato dirigido e um jogo de classificação de cartões, por ordem de importância, que nomeiam eventos de vida diversos. No presente estudo serão apresentados os dados relacionados exclusivamente ao relato espontâneo de eventos de vida positivos e negativos.

As entrevistas foram realizadas individualmente e gravadas em áudio, utilizando-se de gravador e fitas cassete.

\section{Procedimento}

A coleta de dados foi realizada por meio da avaliação individual dos participantes por duas psicólogas e por uma estudante de psicologia, treinadas para a aplicação dos instrumentos utilizados. As avaliações com as mães e as crianças foram realizadas no Centro de Saúde Escola, ou na casa das mesmas, quando assim preferiram; procurou-se manter, nas duas situações, boas condições de privacidade e conforto.

Avaliação com as mães. Após a assinatura do Termo de Consentimento Livre e Esclarecido, procedeu-se à avaliação por meio da Entrevista Clínica Estruturada para o DSM IV, com as mães de ambos os grupos. Em seguida, as mães responderam à Escala Comportamental Infantil A2 de Rutter (ECI). Em geral, as avaliações foram realizadas em uma sessão, com duração de aproximadamente 80 minutos.
Avaliação com as crianças. Na primeira sessão, após um breve rapport, foi realizada a aplicação do teste Matrizes Progressivas Coloridas de Raven e, em seguida, da Escala Infantil Piers-Harris de Autoconceito.

Na segunda sessão, após um breve rapport, realizou-se a Entrevista sobre Eventos Vitais Positivos e Negativos. No que diz respeito ao Relato Espontâneo de Eventos de Vida Positivos e Negativos, a criança era solicitada a comentar, espontaneamente, sobre eventos de vida positivos e negativos que tivesse vivenciado ultimamente, com base nas seguintes instruções:

- Eventos de vida positivos: "Me conte uma coisa boa que aconteceu com você nos últimos dias".

- Eventos de vida negativos: "Me conte uma coisa ruim que aconteceu com você nos últimos dias".

Consideraram-se todos os eventos relatados espontaneamente, sem um limite máximo, e quando as crianças não relatavam nenhum evento, as instruções foram reiteradas, de modo que as crianças relatassem pelo menos um evento positivo e um negativo.

\section{Análise dos dados}

Os instrumentos utilizados neste estudo foram codificados de acordo com as recomendações específicas de cada um deles, os resultados obtidos foram comparados aos dados normativos e realizou-se a comparação entre os dois grupos. Para todas as comparações estatísticas, considerou-se o nível de significância $\mathrm{p} \leq 0,05$.

Os dados da ECI foram pontuados de acordo com as normas de Graminha (1994). Quanto ao escore total, considerou-se a proposição da pontuação de corte (escore $\geq 16$ ) como indicativa da necessidade de encaminhamento para atendimento psicológico. Com base nesse procedimento, identificaram-se dois subgrupos com escores inferiores e superiores à nota de corte.

A codificação da Escala Piers-Harris de Autoconceito foi realizada de acordo com as recomendações do instrumento original. As respostas foram pontuadas em 0 (autoconceito negativo) ou 1 (autoconceito positivo). O escore total corresponde à somatória dos pontos obtidos pela criança.

As entrevistas gravadas com as crianças foram transcritas na íntegra e complementadas por observações relativas a expressões faciais e ao contato com a avaliadora, de modo a facilitar a compreensão dos relatos gravados e a categorização dos temas. Todas as entrevistas foram numeradas aleatoriamente, de maneira a não ser identificado o grupo de origem dos participantes. Aplicou-se, então, um procedimento de categorização dos temas relatados pelas crianças, utilizandose para tal as definições desenvolvidas por Bianchi (2002). Com base em leitura exaustiva, as respostas das crianças foram agrupadas em categorias, de acordo com a frequência e o tipo de evento relatado. Esse procedimento foi realizado por dois avaliadores independentes e os desacordos foram analisados por um terceiro avaliador. Considerou-se como concordância aceitável o acordo simples, de pelo menos $80 \%$. Para a análise dos dados foram consideradas as codificações de consenso para dois avaliadores. 
Com relação aos dados obtidos pela Escala Piers-Harris de Autoconceito, pela Entrevista de Eventos Vitais Positivos e Negativos e pela ECI, os grupos foram comparados por meio do Teste Não Paramétrico U de Mann-Whitney. As comparações relativas aos itens da ECI foram feitas por meio do Teste Exato de Fisher ou do Teste do Qui-quadrado $\left(x^{2}\right)$.

\section{Resultados}

A Tabela 1 apresenta o resultado da avaliação de aspectos comportamentais das crianças, realizada pelas mães a partir da ECI. Mais especificamente, a tabela apresenta a média (e desvio padrão) dos escores parciais - correspondentes a problemas de saúde, hábito e comportamento - e do escore total de cada um dos grupos. Nas comparações entre grupos, observaram-se diferenças estatisticamente significativas tanto em relação ao escore total ( $p \leq 0,02)$, como em relação ao escore da área 'comportamento' $(\mathrm{p} \leq 0,02)$, sendo que as crianças filhas de mães depressivas apresentaram valores superiores (mais problemas de comportamento) aos das crianças filhas de mães sem história psiquiátrica.
Quanto ao escore total (ET), observaram-se ainda diferenças estatisticamente significativas entre G1 e G2 (p $\leq 0,01)$ quando da comparação com a pontuação de corte, a qual caracteriza a necessidade de atendimento psicológico ou psiquiátrico (ET $\geq 16)$. A análise dos resultados obtidos em relação ao estudo normativo mostrou, para G1, uma porcentagem significativamente maior $(80 \%)$ de crianças com resultados superiores à nota de corte, sugestivo da necessidade de cuidados especializados, enquanto que para G2, apenas $40 \%$ das crianças atingiram tal critério.

A Tabela 2 apresenta as porcentagens de ocorrência de cada item específico da área comportamento em que foram observadas diferenças estatisticamente significativas entre os grupos. Os filhos de mães com depressão apresentaram uma porcentagem maior de ocorrência em três dos 21 itens, a saber: Irritável $(p \leq 0,03)$, Desobediente $(p \leq 0,03)$ e Criança Difícil, Complicada $(\mathrm{p} \leq 0,02)$. Nos demais itens, as diferenças entre os grupos não foram estatisticamente significativas.

A Tabela 3 apresenta a média (e desvio padrão) do escore total e escores parciais das subcategorias de autoconceito, obtidos a partir do relato das crianças. Não foram observadas diferenças estatisticamente significativas entre os grupos em relação ao escore total do autoconceito, indicando que
Tabela 1. Média (M) e desvio padrão (DP) dos escores parciais e do escore total, relativos às áreas de saúde, hábito e comportamento, para os grupos G1 e G2.

\begin{tabular}{lccccc}
\hline \multirow{2}{*}{ Áreas } & \multicolumn{2}{c}{ G1 } & \multicolumn{2}{c}{ G2 } & \multirow{2}{c}{ M ( DP) } \\
\hline Saúde & 3.80 & $(1.59)$ & 3.10 & $(2.17)$ & 0,34 \\
Hábito & 3.10 & $(2.00)$ & 2.15 & $(1.73)$ & 0,12 \\
Comportamento & 14.65 & $(6.11)$ & 9.85 & $(5.63)$ & $0.02 *$ \\
\hline Escore Total & 21.55 & $(7.56)$ & 15.10 & $(7.77)$ & $0.02 *$ \\
\hline
\end{tabular}

Nota: G1=crianças filhas de mães com história de depressão; $G 2=$ crianças filhas de mães sem história psiquiátrica; * $p \leq 0,05$ (Teste Não Paramétrico $U$ de MannWhitney).
Tabela 2. Porcentagem (\%) de ocorrência dos itens da área 'comportamento' (dentre um total de 21 itens) com diferenças estatisticamente significativas entre os grupos G1 e G2.

\begin{tabular}{lccc}
\hline Itens & $\begin{array}{c}\text { G1 } \\
(\%)\end{array}$ & $\begin{array}{c}\text { G2 } \\
(\%)\end{array}$ & $\boldsymbol{p}$ \\
\hline Irritável & 75 & 40 & $0.03^{*}$ \\
Desobediente & 65 & 30 & $0.03^{*}$ \\
Criança difícil, complicada & 25 & 0 & $0.02 *$ \\
\hline
\end{tabular}

Nota: G1=crianças filhas de mães com história de depressão; G2=crianças filhas de mães sem história psiquiátrica; * $p \leq 0,05$ (Teste Exato de Fisher; Teste $\chi^{2}$ ).

Tabela 3. Média (M) e desvio padrão (DP) dos escores parciais e do escore total, relativos às subcategorias de autoconceito, para os grupos G1 e G2.

\begin{tabular}{|c|c|c|c|c|c|}
\hline \multirow{2}{*}{$\begin{array}{l}\text { Categorias } \\
\text { Comportamento }\end{array}$} & \multicolumn{2}{|c|}{$\begin{array}{c}\text { G1 } \\
\text { M ( DP) }\end{array}$} & \multicolumn{2}{|c|}{$\begin{array}{c}\text { G2 } \\
M(\text { ( DP) }\end{array}$} & \multirow{2}{*}{$\frac{p}{0.01 *}$} \\
\hline & 12.90 & $(2.43)$ & 14.50 & $(1.05)$ & \\
\hline Status Intelectual & 13.80 & $(3.00)$ & 14.90 & $(1.89)$ & 0,32 \\
\hline Aparência & 9.55 & $(2.96)$ & 10.45 & $(2.54)$ & 0,32 \\
\hline Ansiedade & 8.65 & $(3.75)$ & 9.80 & $(2.12)$ & 0,52 \\
\hline Popularidade & 8.00 & $(2.64)$ & 9.20 & $(2.88)$ & 0,09 \\
\hline Felicidade e Satisfação & 8.40 & $(1.98)$ & 9.10 & $(1.02)$ & 0,41 \\
\hline Total & 61.30 & $(13.16)$ & 67.95 & $(8.68)$ & 0,09 \\
\hline
\end{tabular}

Nota: G1=crianças filhas de mães depressivas; $\mathrm{G} 2=$ crianças filhas de mães sem história psiquiátrica; ${ }^{*} p \leq 0,05$ (Teste Não Paramétrico $U$ de Mann-Whitney). 
as crianças de ambos os grupos fizeram avaliações globais semelhantes de si. Nas comparações das subcategorias de autoconceito, observou-se diferença estatisticamente significativa entre os grupos na subcategoria 'comportamento' $(p \leq 0,01)$, sendo que as crianças filhas de mães depressivas apresentaram um autoconceito menor, relativo a seus comportamentos, que as crianças filhas de mães sem história psiquiátrica. Nas demais subcategorias não foram observadas diferenças estatisticamente significativas.

Destaca-se, ainda, como dados adicionais, que os grupos também foram comparados quanto a cada um dos itens da escala, sendo que apenas um item (Eu odeio escola) diferenciou significativamente os grupos: nesse item, G1 apresentou uma avaliação mais negativa do autoconceito do que G2 $(\mathrm{p} \leq 0,05)$. Quanto ao relato espontâneo de eventos de vida, não foi observada diferença estatisticamente significativa com relação à média $(\mathrm{M})$ de eventos positivos para $\mathrm{G} 1$ $(\mathrm{M}=1,75 ; \mathrm{DP}=1,25)$ e $\mathrm{G} 2(\mathrm{M}=2,00 ; \mathrm{DP}=1,21)$, e também quanto aos eventos negativos para $\mathrm{G} 1(\mathrm{M}=1,90 ; \mathrm{DP}=1,25)$ e $\mathrm{G} 2(\mathrm{M}=2,00 ; \mathrm{DP}=1,02)$.

A Tabela 4 apresenta as porcentagens de relatos incluídos nas categorias de Eventos Positivos, referidos no relato

Tabela 4. Eventos de vida positivos: porcentagem de eventos relatados espontaneamente, em cada categoria, pelas crianças dos grupos G1 e G2.

\begin{tabular}{|c|c|c|c|}
\hline Categorias & $\begin{array}{l}\text { G1 } \\
\%\end{array}$ & $\begin{array}{l}\text { G2 } \\
\%\end{array}$ & $p$ \\
\hline Ganhar presentes & 25 & 20 & 0,70 \\
\hline $\begin{array}{l}\text { Começar ou realizar uma atividade } \\
\text { nova }\end{array}$ & 25 & 0 & $0,01^{*}$ \\
\hline Comemorar aniversários ou festas & 20 & 5 & 0,15 \\
\hline Passear & 15 & 25 & 0,43 \\
\hline $\begin{array}{l}\text { Alguém da família ficar bem de } \\
\text { saúde(física e mental) }\end{array}$ & 15 & 0 & 0,07 \\
\hline Viver coisas boas com a família & 15 & 30 & 0,26 \\
\hline Conviver com os amigos & 15 & 15 & 1,00 \\
\hline Brincar & 10 & 30 & 0,11 \\
\hline $\begin{array}{l}\text { Ir bem na escola (notas, amigos, } \\
\text { professores) }\end{array}$ & 10 & 30 & 0,11 \\
\hline Mudar de escola ou casa & 5 & 5 & 1,00 \\
\hline Descansar & 0 & 20 & $0,03 *$ \\
\hline $\begin{array}{l}\text { Melhorar situação econômica e/ou } \\
\text { social da família }\end{array}$ & 0 & 5 & 0,31 \\
\hline
\end{tabular}

Nota: G1=crianças filhas de mães com história de depressão; G2=crianças filhas de mães sem história psiquiátrica; ${ }^{*} p \leq 0,05$ (Teste Não Paramétrico $U$ de Mann-Whitney). espontâneo das crianças. Observou-se diferença estatisticamente significativa em dois dos 16 eventos positivos relatados espontaneamente. As crianças de G1 apresentaram, comparativamente às crianças de G2, uma maior porcentagem de relatos de eventos relacionados à categoria Começar ou realizar uma atividade nova $(\mathrm{p} \leq 0,01)$. Quanto à categoria Descansar, as crianças de G1 mostraram uma menor porcentagem de relatos de eventos relacionados a essa categoria $(\mathrm{p} \leq 0,03)$.

A Tabela 5 apresenta as porcentagens de relatos incluídos nas categorias de Eventos Negativos, referidos no relato espontâneo das crianças. Observou-se que apenas uma categoria, dentre as 15 categorias de eventos negativos, diferenciou

Tabela 5. Eventos de vida negativos: porcentagem de eventos relatados espontaneamente, em cada categoria, pelas crianças dos grupos G1 e G2.

\begin{tabular}{|c|c|c|c|}
\hline Categorias & $\begin{array}{l}\text { G1 } \\
\%\end{array}$ & $\begin{array}{l}\text { G2 } \\
\%\end{array}$ & $p$ \\
\hline $\begin{array}{l}\text { Alguém da família ficar doente ou } \\
\text { sofrer acidente }\end{array}$ & 30 & 15 & 0,26 \\
\hline $\begin{array}{l}\text { Acontecer brigas ou desentendimen- } \\
\text { tos em casa }\end{array}$ & 25 & 15 & 0,43 \\
\hline $\begin{array}{l}\text { Ficar afastado de familiar querido ou } \\
\text { de alguém que gosta }\end{array}$ & 20 & 10 & 0,38 \\
\hline $\begin{array}{l}\text { Não poder fazer alguma coisa que } \\
\text { queria muito }\end{array}$ & 15 & 5 & 0,29 \\
\hline $\begin{array}{l}\text { Ter problemas na escola (notas, ami- } \\
\text { gos, professores) }\end{array}$ & 15 & 10 & 0,63 \\
\hline $\begin{array}{l}\text { Acontecer brigas ou desentendimen- } \\
\text { tos dos pais }\end{array}$ & 15 & 10 & 0,63 \\
\hline Brigar ou se desentender com amigos & 10 & 15 & 0,63 \\
\hline Sentir medo, ficar inseguro & 10 & 20 & 0,38 \\
\hline Passar por situações de violência & 10 & 15 & 0,63 \\
\hline $\begin{array}{l}\text { Perder alguém querido - morte de } \\
\text { alguém }\end{array}$ & 10 & 15 & 0,63 \\
\hline Ficar doente ou se machucar & 5 & 30 & $0,04 *$ \\
\hline Ser ridicularizado ou desprezado & 5 & 5 & 1,00 \\
\hline Receber advertências e punições & 5 & 5 & 1,00 \\
\hline $\begin{array}{l}\text { Família passar por dificuldades eco- } \\
\text { nômicas e/ou sociais }\end{array}$ & 0 & 5 & 0,31 \\
\hline $\begin{array}{l}\text { Acontecer maus tratos, morte ou } \\
\text { afastamento de animais }\end{array}$ & 0 & 5 & 0,31 \\
\hline
\end{tabular}

Nota: G1=crianças filhas de mães com história de depressão; G2=crianças filhas de mães sem história psiquiátric; ${ }^{*} p \leq 0,05$ (Teste Não Paramétrico $U$ de Mann-Whitney). 
significativamente os grupos, sendo esta referente a Ficar doente ou se machucar, na qual G1 relatou menor número de eventos comparativamente à $\mathrm{G} 2(\mathrm{p} \leq 0,05)$.

\section{Discussão}

Destaca-se, como resultado principal do presente estudo, que as crianças que convivem com a depressão materna foram apontadas por suas mães como tendo mais problemas de comportamento, comparativamente às crianças filhas de mães sem história de depressão. Esse achado está de acordo com os relatos de Najman e cols. (2001) e Boyle e Pickles (1997) que apontaram uma associação significativa entre depressão materna e problemas de comportamento por parte das crianças.

A análise dos itens específicos da área 'comportamento' mostrou que as crianças que convivem com a depressão materna se diferenciaram das crianças do grupo de comparação nos itens irritável, desobediente e difícil, e complicada que caracterizam indicadores de alterações do humor. Pode-se compreender tal resultado tendo por base as afirmativas de Goodman e Gotlib (1999) que apontam que as vulnerabilidades das crianças que convivem com a depressão materna podem emergir nos domínios afetivo, manifesto por dificuldades na regulação emocional, e comportamental, evidenciado por habilidades sociais inadequadas e dificuldades de controle de impulsos. Os referidos autores, em concordância com Cummings (1995), indicaram que a convivência com mães depressivas expõe as crianças a manifestações próprias do quadro depressivo, como irritabilidade e desânimo, as quais podem favorecer manifestações afetivas negativas por parte das crianças, além de comprometer a aprendizagem de habilidades de regulação emocional.

A constatação de mais dificuldades comportamentais por parte das crianças que convivem com a depressão materna remete ao relato de Silk e cols. (2006) que referem que as mães depressivas, por apresentarem limitações em suas próprias habilidades de regulação emocional, não dispõem dos recursos necessários para ensinar tais habilidades aos seus filhos, ou ainda para reforçar as manifestações afetivas e os meios mais adaptativos de modulação do estresse por parte destes. Esses autores relataram, ainda, que as dificuldades de comportamento das crianças expostas à depressão materna refletem as influências da aprendizagem social e da falta de recursos de suas mães para a modelagem de comportamentos mais adaptativos. De modo semelhante, Gross (1994) destaca que as mães com depressão apresentam menos disposição para a colocação de limites para os seus filhos, o que dificulta a aprendizagem de normas sociais, as quais exigem controle e regulação emocional.

Nesse sentido, a presença de dificuldades comportamentais por parte de tais crianças pode estar refletindo as experiências de serem frequentemente criticadas ou reforçadas por suas próprias visões negativas de mundo, pois segundo Goodman e Gotlib (1999), é bastante frequente que as crianças que convivem com a depressão materna apresentem afetos, comportamentos e cognições muito semelhantes aos de suas mães depressivas, sugerindo uma visão negativa partilhada na convivência cotidiana.
No presente estudo, observou-se que uma maior porcentagem de mães com história de depressão identificou a necessidade de cuidados especializados de saúde mental para os seus filhos, caracterizando assim a percepção de que suas crianças estão enfrentando dificuldades. Esse resultado é concordante com o relatado por Weissman e Jensen (2002), que identificaram que crianças, filhas de mulheres com depressão, tendem a apresentar maiores índices de busca por tratamento para problemas de saúde mental, quando comparadas com crianças filhas de pais sem história psiquiátrica.

Tais crianças estão sendo vistas por suas mães como apresentando dificuldades que podem prejudicar a socialização com seus pares e a adaptação ao meio, portanto estão experimentando dificuldades quanto à tarefa de socialização, própria do período escolar. Esses achados vão ao encontro do proposto por Luoma e cols. (2001), que constataram a presença de baixa competência social e de baixo funcionamento adaptativo nas crianças que convivem com as manifestações depressivas maternas.

Sob a perspectiva da psicopatologia do desenvolvimento, as dificuldades para o cumprimento das tarefas de desenvolvimento próprias da idade caracterizam desadaptação, apontando para a necessidade de suporte social, de ajuda para o enfrentamento do estresse e de monitoramento do comportamento (Sameroff \& Fiese, 2005). Pode-se pensar que as mães com depressão, ao identificarem as dificuldades e as necessidades de ajuda às suas crianças, de certo modo, estão percebendo a necessidade de suporte, denotando preocupação e cuidado com seus filhos. Tal hipótese tem apoio também na não diferenciação dos grupos nas áreas Saúde e Hábitos, sugerindo que as mães de G1 estão dispensando os recursos e cuidados necessários para o desenvolvimento físico dessas crianças, o que reforça o foco nas dificuldades comportamentais relacionadas à adaptação e ao desenvolvimento de habilidades sociais e emocionais.

Um outro ponto a ser discutido relaciona-se aos dados controversos da literatura quanto à validade dos relatos das mães com depressão sobre os comportamentos dos filhos, sugerindo que essas tenderiam a ter uma visão mais negativa dos filhos, e à percepção de mais problemas de comportamento do que eles realmente apresentam. No estudo em questão, além da mãe como informante, tomou-se o relato da própria criança quanto ao autoconceito e aos eventos de vida como fonte de informação independente.

Considerando a variável autoconceito, observou-se que as crianças, a despeito de serem filhas ou não de mães depressivas, percebem-se de forma semelhante quanto à avaliação global. Dada a ausência de dados normativos para a Escala Infantil Piers-Harris de Auto-Conceito no Brasil, tal dado nos faz supor que essa percepção é sugestiva do predomínio de uma avaliação positiva de si, já que ela foi apresentada também pelo grupo de comparação (G2). Quanto às subcategorias, observou-se que as crianças que convivem com a depressão materna relataram um autoconceito mais negativo na área relacionada ao comportamento que as crianças que convivem com mães sem história psiquiátrica. Tal achado faz pensar na possibilidade de tais crianças estarem internalizando a opinião de suas mães sobre seu comportamento, o que vai ao encontro das proposições de Harter (1996) ao 
afirmar que a construção do autoconceito está associada às experiências sociais e às expectativas externas.

Ao se analisar o autoconceito com base em itens da subcategoria 'comportamento', observou-se que os grupos diferiram em um único item (Odeio escola). As crianças que convivem com a depressão materna parecem ter uma visão mais negativa relacionada à escola, o que está diretamente relacionado à tarefa de produtividade, típica do período de desenvolvimento em que essas crianças se encontram. Nesse período de desenvolvimento, segundo Marturano e Loureiro (2003), o desempenho acadêmico e o ajustamento escolar se revestem de importância como tarefas esperadas de socialização. Para alcançarem as aquisições próprias da idade, as crianças buscam apoio e orientação em seus pais, e, nesse sentido, em concordância com as proposições de Goodman e Gotlib (1999), pode-se pensar que as mães depressivas estão com dificuldades de prover tais cuidados aos seus filhos. Verificando-se as características demográficas das mães e as condições de configurações das famílias, não foram observadas diferenças de escolaridade materna ou de possibilidade de suporte que possam ter contribuído para esse resultado, o que, de certo modo, reforça a influência da depressão materna para o comportamento da criança.

A maior incidência de eventos estressores no cotidiano das crianças que convivem com a depressão materna, como apontado por Goodman e Gotlib (1999), não foi constatada no estudo em questão, quando da avaliação dos eventos de vida relatados espontaneamente pelas crianças de ambos os grupos. Tal resultado sugere semelhança com relação à diversidade de experiências de vida a que as crianças estão expostas em seu cotidiano e à percepção de eventos de vida. Esses dados se contrapõem à hipótese de negligência por parte das mães depressivas e chama a atenção para a possibilidade da maior influência da depressão materna, para as crianças do estudo em questão, estar relacionada à dificuldade de adequação quanto ao controle dos impulsos.

Outros pontos a serem analisados são referentes aos eventos positivos de vida, que ocorreram com diferenças significativas entre os grupos, a saber: Começar ou realizar uma atividade nova e Descansar. A maior porcentagem de relato de situações relacionadas a Começar ou realizar uma atividade nova, por parte das crianças que convivem com a depressão materna, incluindo atividades como esportes, dança, música e computação, pode indicar o interesse dessas crianças por tarefas que envolvem aprendizado, o que pode favorecer a aquisição de novas competências, como o esperado nesse período do desenvolvimento. Tal dado é sugestivo da possibilidade de tais atividades serem potencialmente úteis para as práticas de saúde mental direcionadas a essas crianças.

Observou-se uma menor porcentagem de situações relacionadas a Descansar, o que sugere que as crianças que convivem com a depressão materna estão tendo um estilo de vida ativo, buscando se envolver com atividades como forma de ampliar as experiências do seu cotidiano. Ao se analisar tais dados, pode-se supor que essas crianças estão apresentando indicadores de habilidades para superarem as adversidades a que estão expostas, buscando o engajamento em diversas atividades que ampliam as oportunidades de aprendizagem e de interação, o que, segundo as proposições de Wright e
Masten (2006) e Yunes e Szymanski (2001), caracterizam a presença de mecanismos de proteção que favorecem a superação das situações adversas.

Analisando-se, ainda, os eventos negativos de vida, observou-se que as crianças que convivem com a depressão materna relataram menor número de eventos relacionados a Ficar doente ou se machucar, quando comparadas às crianças que convivem com mães sem história psiquiátrica. Tal achado pode sugerir que tais crianças, filhas de mães com depressão, têm menor preocupação com sua saúde, ou ainda, que não se detêm muito nessas dificuldades.

A semelhança entre os eventos de vida relatados espontaneamente pelas crianças de ambos os grupos sugere que a depressão materna pode não estar influenciando o cotidiano das crianças em termos de rotinas e cuidados diários, mas sim, pode estar atuando na aprendizagem do controle sobre os afetos e, dessa forma, influenciando o seu comportamento. Tal achado reforça a hipótese da aprendizagem social, segundo a qual as crianças internalizam comportamentos, cognições e afetos negativos expressos por suas mães.

Verificou-se, com base nas duas fontes de informações (mães e crianças), a presença de dificuldades comportamentais para as crianças que convivem com a depressão materna, o que é sugestivo da necessidade de intervenções de saúde mental.

\section{Considerações Finais}

Conclui-se, dentro dos limites do delineamento transversal adotado, que a convivência com a depressão materna mostrou-se associada a problemas de comportamento para crianças em idade escolar, segundo o relato das mães e a percepção das crianças.

A influência da depressão no relato que as mães fazem sobre o comportamento de seus filhos tem sido objeto de questionamento na literatura. A utilização de duas fontes diferentes de informação sobre o comportamento da criança, no caso as mães e as próprias crianças, constitui-se em ponto positivo do delineamento em questão. Destaca-se, ainda, que a condição de remissão de sintomas há pelo menos seis meses, no caso das mães, garantiu certa homogeneidade de condições para a avaliação do comportamento das crianças, minimizando a influência de diferentes níveis atuais de sintomatologia depressiva sobre a percepção do comportamento dos filhos.

Ressalta-se a necessidade de novos estudos que busquem ampliar a compreensão sobre as implicações da depressão materna para o comportamento infantil, utilizando amostras maiores e outras fontes de informações externas à família, como, por exemplo, os professores, de modo a investigar o comportamento das crianças fora do ambiente familiar.

Os dados do presente estudo reafirmam o impacto negativo da depressão materna para o comportamento das crianças, caracterizando-se como condição de vulnerabilidade que requer intervenções de saúde mental, com o objetivo de promover mecanismos de proteção ao desenvolvimento infantil. 


\section{Referências}

Angelini, A. L., Alves, I. C., Custódio, E. M., Duarte, W. F., \& Duarte, J. L. (1999). Manual das Matrizes Progressivas Coloridas de Raven: escala especial. São Paulo: Centro Editor de Testes e Pesquisas em Psicologia.

Bianchi, S. H. (2002). Eventos de vida, auto-eficácia e autoconceito de crianças com dificuldades comportamentais. Dissertação de Mestrado, Universidade de São Paulo, Ribeirão Preto.

Boyle, M. H., \& Pickles, A. R. (1997). Influence of maternal depressive symptoms on ratings of childhood behavior. Journal of Abnormal Child Psychology, 25, 399-412.

Cummings, E. M. (1995). Security, emotionality and parental depression: A commentary. Developmental Psychology, 31, 425-427.

Chilcoat, H. D., \& Breslau, N. (1997). Does psychiatric history bias mothers' reports? An application of a new analytic approach. Journal of the American Academy of Child and Adolescent Psychiatry, 36, 971-979.

Del-Ben, C. M., Vilela, J. A. A., Crippa, J. A. S., Hallak, E. C., Labate, C. M., \& Zuardi, A. W. (2001). Confiabilidade da "Entrevista Clínica Estruturada para o DSM-IV - Versão Clínica" traduzida para o português. Revista Brasileira de Psiquiatria, 23, 156-159.

Dell'Aglio, D. D. (2000). O Processo de coping, institucionalização e eventos de vida em crianças e adolescentes. Tese de Doutorado, Universidade Federal do Rio Grande do Sul, Porto Alegre.

Goodman, S. H., \& Gotlib, I. H. (1999). Risk for psychopathology in the children of depressed mothers: A developmental model for understanding mechanisms of transmission. Psychological Review, 106, 458-490.

Graminha, S. S. V. (1994). A Escala Comportamental Infantil de Rutter A2: estudos de adaptação e fidedignidade. Estudos de Psicologia, 11, 34-42.

Gross, D. A. (1994). Children of the mentally ill. Em B. S Jonhson (Org.), Child, adolescent and family psychiatric nursing (pp. 101-113). New York: J. B. Lippincott.

Harter, S. (1996). Historical roots of cotemporary issues involving self-concept. Em B. A. Bracken (Org). Handbook of self-concept developmental, social and clinical considerations (pp. 1-37). New York: John Wiley \& Sons.

Jacob, A. V. (2001). O desempenho escolar e suas relações com autoconceito e autoeficácia. Tese de Doutorado, Universidade de São Paulo, Ribeirão Preto.

Kendler, K. S., \& Prescott, C. A. (1999). A population-based twin study of lifetime major depression in men and women. Archives of General Psychiatry, 56, 39-44.

Kinsman, A. M., \& Wildman, B. G. (2001). Mother and child perceptions of child functioning: Relationship to maternal distress. Family Process, 40, 163-172.

Langrock, A. M., Compas, B. E., Keller, G., Merchant, M. J., \& Copeland, M. E. (2002). Coping with the stress of parental depression: Parent's reports of children's coping, emotional and behavioral problems. Journal of Clinical Child and Adolescent Psychology, 31, 312-324.

Luoma, I., Tamminem, T., Kaukonem, M. P., Laippala, P., Puura, K., Salmelin, R., \& Almqvist, F. (2001). Longitudinal study of maternal depressive symptoms and child well-being. Journal of the American Academy of Child and Adolescent Psychiatry 40, 1367-1374.
Mari, J. J., \& Jorge, M. R. (2002). Epidemiologia dos transtornos mentais. Em J. J. Mari, K. D. Razzouk, M. F. T. Peres \& J. A. Del Porto (Orgs.), Guias de Medicina Ambulatorial e HospitalarUnifesp/Escola Paulista de Medicina - Psiquiatria (pp. 1-6). São Paulo: Manole.

Marturano, E. M., \& Loureiro, S. R. (2003). O desenvolvimento socioemocional e as queixas escolares. Em A. Del Prette \& Z. A. P. Del Prette (Orgs.), Habilidades sociais, desenvolvimento e aprendizagem: questões conceituais, avaliação e intervenção (pp. 259-291). Campinas: Editora Alínea.

Najman, J. M., Williams, G. M., Nickles, J., Spence, S., Bor, W., O’Callaghan, M., Le Brocque, R., Andersen, M. J., \& Shuttlewood, G. J. (2001). Bias influencing maternal reports of child behavior and emotional state. Social Psychiatry and Psychiatric Epidemiology, 36, 186-194.

Organização Mundial de Saúde (1993). Classificação de Transtornos Mentais e de Comportamento da CID-10. (D. Caetano, Trad) Porto Alegre: Artes Médicas. (Trabalho original publicado em 1992)

Pereira, A. M. S. (2001). Resiliência, personalidade, stress e estratégias de coping. Em J. Tavares (Org.), Resiliência e educação (pp.77-94). São Paulo: Cortez.

Piers, E. V., \& Harris, D. B. (1984). Piers-Harris Children's SelfConcept Scale - Revised Manual. Los Angeles: Counselor Psychological Tests, Western Psychological Services.

Sameroff, A. J., \& Fiese, B. H. (2005). Models of development and developmental risk. Em C. H. Zeanah, Jr. (Org.), Handbook of infant mental health (pp. 3-19). Springer, New York: Guilford Press.

Silk, J. S., Shaw, D. S., Skuban, E. M., Oland, A. A., \& Kovacs, M. (2006). Emotion regulation strategies in offspring of childhoodonset depressed mothers. Journal of Child Psychology and Psychiatry. 47, 69-78.

Weissman, M. M., \& Jensen, P. (2002). What research suggests for depressed women with children. Journal of Clinical Psychiatry, 63, 641-647.

Wright, M. O., \& Masten, A. S. (2006). Resilience process in development: Fostering positive adaptation in the context of adversity. Em S. Goldstein \& R. B. Brooks (Orgs.), Handbook of resilience in children. (pp. 17-37). Springer, New York: Business Media.

Yunes, M. A. M., \& Szymanski, H. (2001). Resiliência: noção, conceitos afins e considerações críticas. Em J. Tavares (Org.), Resiliência e educação (pp. 13-42). São Paulo: Cortez. 